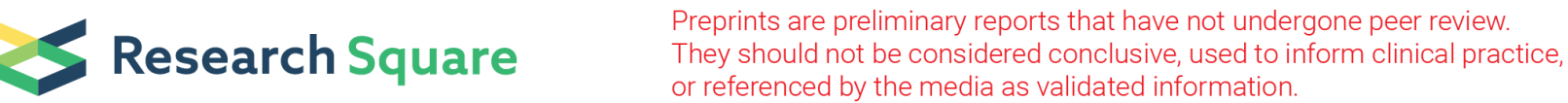

\section{Pathogen Infection Recovery Probability (PIRP) Versus Proinflammatory Anti-Pathogen Species (PIAPS) Levels: Modelling and Therapeutic Strategies}

\author{
Sam-Shajing Sun ( $\nabla$ ssun@nsu.edu ) \\ Norfolk State University https://orcid.org/0000-0002-4905-227X
}

\section{Method Article}

Keywords: COVID-19, pathogen infection, anti-pathogen species (APS), proinflammatory anti- pathogen species (PIAPS), hyperinflammatory disorders, cytokine storm, modelling, pathogen infection recovery probability (PIRP), optimal PIRP, saturation/equilibrium levels of APS/PIAPS

Posted Date: March 13th, 2020

DOI: https://doi.org/10.21203/rs.3.rs-17318/v1

License: (c) (1) This work is licensed under a Creative Commons Attribution 4.0 International License. Read Full License

Version of Record: A version of this preprint was published at International Journal of Medical Science and Clinical invention on September 5th, 2020. See the published version at https://doi.org/10.18535/ijmsci/v7i08.06. 


\section{Abstract}

Current new COVID-19 viruses have already spread in multiple countries and have resulted in over one hundred thousand human infections and over three thousand human deaths worldwide. The possibility of a global pandemic is growing. It appears most of the infection resulted deaths are mainly due to dysfunctions or failures of the lung or multiple organs that could be attributed to host immune system's hyperinflammatory disorders. In this work, a model is proposed to correlate the Pathogen Infection Recovery Probability (PIRP) versus Proinflammatory Anti-Pathogen Species (PIAPS) levels within a host unit, where a maximum PIRP is exhibited when the PIAPS levels are equal to or around PIAPS equilibrium levels at the pathogen elimination or clearance onset. Based on this model, rational or effective therapeutic strategies at right stages or timing, with right type of agents and right dosages, can be designed and implemented that are expected to effectively achieve maximum PIRP or reduce the mortality.

\section{Full Text}

Current COVID-19 viruses have already spread in multiple countries around the globe and have resulted in over one hundred thousand human infections and over three thousand human deaths [1-2]. In addition to loss of human life, social and economic losses or effects could be significant. A number of earlier global pandemics occurred in human history can be attributed to pathogen infections [3]. Though there are differences among different pathogen induced infections, there were certain similarities among all pathogen infections. The pathogens here include viruses (such as the new COVID-19 virus), bacteria, or certain substances that can trigger or initiate a host immune system responses resulting in the production (clonal expansion) of anti- pathogen species (APS), including both non-inflammatory and proinflammatory anti-pathogen species (PIAPS). PIAPS include many "double-edged sword" species such as certain white blood cells or related species [4-5], antibodies [6], cytokines [7-12, 18], etc. "Double-edged sword" refers to certain PIAPS that not only attack the pathogens but also attack host healthy cells or tissues $[4-12,18]$.

Pathogen infection modeling can be very useful for understanding the infection mechanisms and processes, and for preventive or therapeutic strategies. However, most of the existing modeling works are mainly focusing on multiple host infection and transmittance statistics over time [13-17], very few modeling work provide insights on pathogen infection recovery probability (PIRP) over anti-pathogens species (APS), particularly over proinflammatory anti- pathogen species (PIAPS) that is the focus of this work.

A pathogen infection could result in pathogen un-controlled growth if the host immune system is deficient (like in certain immune deficiency related syndromes). In a normal or healthy host, as illustrated in Figure 1 , the pathogen infection at time $\mathbf{t} 0$ shall trigger an efficient and normal growth (clonal expansion) of immune system generated anti-pathogen species (APS, including PIAPS) and ideally shall result in pathogen being eliminated or cleared at $\boldsymbol{t e}$ (Figure 1) [13]. Once the pathogen is eliminated or cleared by 
the APS/PIAPS at $\boldsymbol{t} \boldsymbol{e}$, the APS/PIAPS shall reach their equilibrium or saturation level $\boldsymbol{x} \boldsymbol{e}$. Certain APS/PIAPS shall remain at the xelevel for certain period of time so the same pathogen infection can be prevented or inhibited, though APS/PIAPS equilibrium level slow decay in long period of time are expected [13-17].

In a number of autoimmune or hyperinflammatory disorders such as cytokine release syndromes (CRS), macrophage activation syndromes (MAS), certain PIAPS grow out of control even after te where the pathogen has been eliminated or cleared. The excessive PIAPS are believed to attack or damage healthy cells or tissues resulting in critical organ dysfunctions or failures $[2,4-12,18]$. For this reason, a bell shaped normal distribution function $Y$ is proposed here to model the Pathogen Infection Recovery Probability (PIRP, counter to the mortality) versus the PIAPS levels $\boldsymbol{x}$ (shown in Figure 2) and is exhibited in equation (1):

$$
Y=\boldsymbol{\beta} \exp \left[-(x-x e)^{2} / a\right]
$$

where a parameter is proportional to the PIRP distribution peak full width at half maximum (FWHM) that affects the PIAPS level range width around PIRP maximum. During this range, PIAPS levels can significantly elevate PIRP as compared to other PIAPS range where PIRP remains relatively low. $\boldsymbol{\beta}$ parameter represents a coupling factor of PIRP versus PIAPS levels, reflecting how significant or effective PIAPS level affects PIRP.

Based on this model, the PIRP-PIAPS curve are divided into two stages: 1) Stage I or the PIRP rising stage corresponding to pathogen/APS evolution time period between to to te in Figure 1: The PIRP of the pathogen infected host starts to rise as the immune response generated APS/PIAPS levels are growing (solid blue line) and eventually approaching an equilibrium or saturation level $\boldsymbol{x e}$ where the pathogen are being eliminated or cleared. 2) Stage II or the PIRP descending stage: The PIAPS level further increases beyond their equilibrium or saturation level xeas represented by the long dashed blue line (such as in many autoimmune or hyperinflammatory disorders) $[2,4-12,18]$, the PIRP starts to descends, presumably due to excessive or extra PIAPS starting to attack or damage the healthy tissues or organs. Eventually the PIRP could descend to a minimum level (resulting in host death) when excessive PIAPS growth is out of control (dysregulated) and results in critical organ dysfunctions, such as in the cases of cytokine storms $[2,7-12,18]$.

Based on this model, the general therapeutic strategies for minimizing mortality is to achieve and/or sustain maximum PIRP as following: 1) In the stage I or the PIRP rising stage, if the host has a normal immune response to the pathogen infection, the APS/PIAPS are expected to grow efficiently toward an equilibrium or saturation level $\boldsymbol{x e}$ where the pathogens are eliminated or cleared. In this stage and situation, no therapeutic treatments are needed except certain standard host recovery support or assistance. However, if host's APS/PIAPS do not respond to the infection (such as the host has certain immune deficiency syndromes), or the APS/PIAPS growth rates are far slower than the pathogen growth rate, than either pathogen inhibitors/suppressors (if available) or APS/PIAPS boosters/enhancers (e.g., 
certain white blood cell therapies, immunoglobin therapies, as well as therapies utilizing plasma and antibodies obtained from the convalescent patients) may be administered, but the APS particularly PIAPS booster/enhancer applications must be at the right time (i.e., in the PIRP rising stage I), right type (i.e., APS/PIAPS boosters/enhancers instead of inhibitors/suppressors), and at the right dosages (e.g., total APS/PIAPS levels should be equal or close to their equilibrium or saturation level $\boldsymbol{x e}$ ). 2) In the stage II or the PIRP descending stage when the PIAPS level of the infected host is excessive or its growth is out of control, pathogen inhibitors/suppressors may not be necessary at this time (unless the coupling of the host generated PIAPS to the pathogen is very poor, i.e., pathogen level are still largely positive even with excessive PIAPS level). The most critical or essential therapeutic task in the PIRP descending or stage II shall be to stop the further growth and control or modulate the PIAPS levels nearby their equilibrium levels $\boldsymbol{x}$ e. Though a number of PIAPS control or modulation efforts have been reported in recent years [412], the timing, type, and dosages of PIAPS suppressors or modulators must be carefully monitored and controlled based on Figures 1 and 2, as APS/PIAPS over- suppression could result in a vulnerability of host re-infection and un-controlled pathogen growth. Precise, fast, convenient, and reliable protocols or methods of measuring and monitoring pathogen and targeted PIAPS levels are essential not only to validate this model, but to utilize this model for safe and effective therapeutic treatments of the infected hosts.

As an example, COVID-19 infection virus levels via RNA analysis at appropriate time intervals may be utilized to determine the virus elimination or clearance onset time te and the corresponding APS/PIAPS saturation levels $\boldsymbol{x} e$. An approach on controlling dysregulated interferon INF-1 production in COVID-19 infection [18] could be potentially very useful for validating this model, however, according to this model, the interferon INF-1 level control should be done after $\boldsymbol{t} e$ and the level should not be over compressed well below $x e$. Finally, multiple host units may be utilized to obtain average values of all four parameters of this model $(\boldsymbol{t} \boldsymbol{e}, \boldsymbol{X e}, \boldsymbol{a}, \boldsymbol{\beta})$ for a particular host group, and the average values may be applied to therapeutic treatments of an individual host that is same or similar to the members of the group.

In summary, a bell shaped normal distribution function is proposed to model the Pathogen Infection Recovery Probability (PIRP) versus Proinflammatory Anti-pathogen Species (PIAPS) level in a pathogen infected host. Based on this model, therapeutic strategies should be based on two stages: In the first or PIRP rising stage, treatments may not be necessary for most normal hosts as PIRPs are expected to grow and remain at the maximum due to APS/PIAPS growing to and remain at the equilibrium levels $\boldsymbol{x} e$ upon passing the pathogen elimination onset. Hosts with weak or deficient anti-pathogen immune responses may need either pathogen suppressors or APS/PIAPS boosters, however, timing, type, and dosages of pathogen suppressors or APS/PIAPS boosters are critical. In the second or the PIRP descending stage II due to PIAPS excessive levels, it is essential to control the PIAPS around the equilibrium level $\boldsymbol{x} e$. Again, timing, types, and dosages of therapeutic treatments are extremely critical depending on the PIRP stages and on pathogen/APS/PIAPS levels. Precise and timely monitoring and controls of both pathogen and PIAPS levels are essential in order to fully characterize and utilize this model. Increased PIRP or reduced mortality could be potential outcome if this model is further developed, well characterized, and implemented upon carefully designed and controlled clinical trials. 


\section{Declarations}

\section{Acknowledgement}

The author wishes to thank his child's allergy specialist Dr. Kelly Maples for helpful discussions on cytokines/chemokines related hyper-inflammations in pathogen infections and food allergies, and to thank biochemistry Professor/Dr. Joseph Hall for helpful discussions on pathogen/anti- pathogens. The author particularly wishes to acknowledge and thank his brother Mr. Honggang Sun on insightful discussions about "Happy Medium" doctrine of the Confucius philosophy that was established and taught in China for thousands of years.

\section{Conflict of Interest}

The authors declare no any conflict of interest for publishing this article.

\section{References}

1. COVID-19 Situation Report-49, World Health Organization (WHO), March 9, 2020. (https://www.who.int/emergencies/diseases/novel-coronavirus-2019)

2. Chen Y, Liu Q, Guo D. "Emerging coronaviruses: Genome structure, replication, and pathogenesis". J Med Virol., 92:418-423 (2020) (https://doi.org/10.1002/jmv.25681)

3. https://en.wikipedia.org/wiki/Pandemic

4. Leslie, , "The body's dangerous defenders", Science 367 (6482), 1067-1069 (2020) (DOI: 10.1126/science.367.6482.1067).

5. Vandenhaute, J., Wouters, C., Matthys, P., "Natural Killer Cells in Systemic Autoinflammatory Diseases: A Focus on Systemic Juvenile Idiopathic Arthritis and Macrophage Activation Syndrome", Immunol., 15 January 2020. (https://doi.org/10.3389/fimmu.2019.03089)

6. Wesemann , Nagler, C., "Origins of Peanut Allergy-Causing Antibodies”, Science 367 (6482), 10721073 (2020) (DOI: 10.1126/science.aba8974).

7. Hay, K., et al., "Kinetics and biomarkers of severe cytokine release syndrome after CD19 chimeric antigen receptor-modified T-cell therapy", BLOOD, 130, 21 (2017) (DOI 10.1182/blood-2017-06, 793141)

8. Liu, , Zhou, Y., Yang, Z., "The cytokine storm of severe influenza and development of immunomodulatory therapy", Cellular \& Molecular Immunology, 13, 3-10 (2016) (doi:10.1038/cmi.2015.74)

9. Tisoncik JR, Korth MJ, Simmons CP, Farrar J, Martin TR, Katze "Into the eye of the cytokine storm", Microbiol Mol Biol Rev. 76(1):16-32 (2012) (doi:10.1128/MMBR.05015-11)

10. Luo C, Liu J, Qi W, et al. "Dynamic analysis of expression of chemokine and cytokine gene responses to H5N1 and H9N2 avian influenza viruses in DF-1 cells". Microbiol Immunol., 62(5):327-340 (2018) (doi:10.1111/1348-0421.12588). 
11. Staedtke, V., et al., "Disruption of a self-amplifying catecholamine loop reduces cytokine release syndrome", Nature, 564, 273 (2018) (https://doi.org/10.1038/s41586- 018-0774-y).

12. Teijaro, J., Walsh, K., Rice, S., Rosen, H., Oldstone, M., "Mapping the innate signaling cascade essential for cytokine storm during influenza virus infection". Proceedings of the National Academy of Sciences, 111 (10) 3799-3804 (2014) (DOI: 10.1073/pnas.1400593111)

13. Gulbudak, $\mathrm{H}$ and Browne, C., "Infection severity across scales in multi-strain immuno-epidemiological Dengue model structured by host antibody level", arXiv:1912.08305 [q-bio.PE], December 17,

14. Barbarossa, M. and Rost, G., "Immuno-epidemiology of a population structured by immune status: a mathematical study of waning immunity and immune system boosting", arXiv:1411.3195 [math.DS], November 14, 2014.

15. Uekermann, F., Sneppen, K., "Cross-immunization model for the extinction of old influenza strains". Sci Rep., 6, 25907 (2016). (https://doi.org/10.1038/srep25907).

16. Siettos, C. \& Russo, L., "Mathematical modeling of infectious disease dynamics", Virulence, 4:4, 295306 (2013) (DOI:10.4161/viru.24041)

17. Zhu X, Kranse R, Bul M, Bangma CH, Schröder FH, Roobol MJ. “Overestimation of prostate cancer mortality and other-cause mortality by the Kaplan-Meier method". Can J Urol. 20(3):6756-6760 (2013) (PMID:23783043).

18. Deng, X., Yu , X., Pei, J., "Regulation of interferon production as a potential strategy for COVID-19 treatment", arXiv:2003.00751 [q-bio.MN], March 2.

\section{Figures}




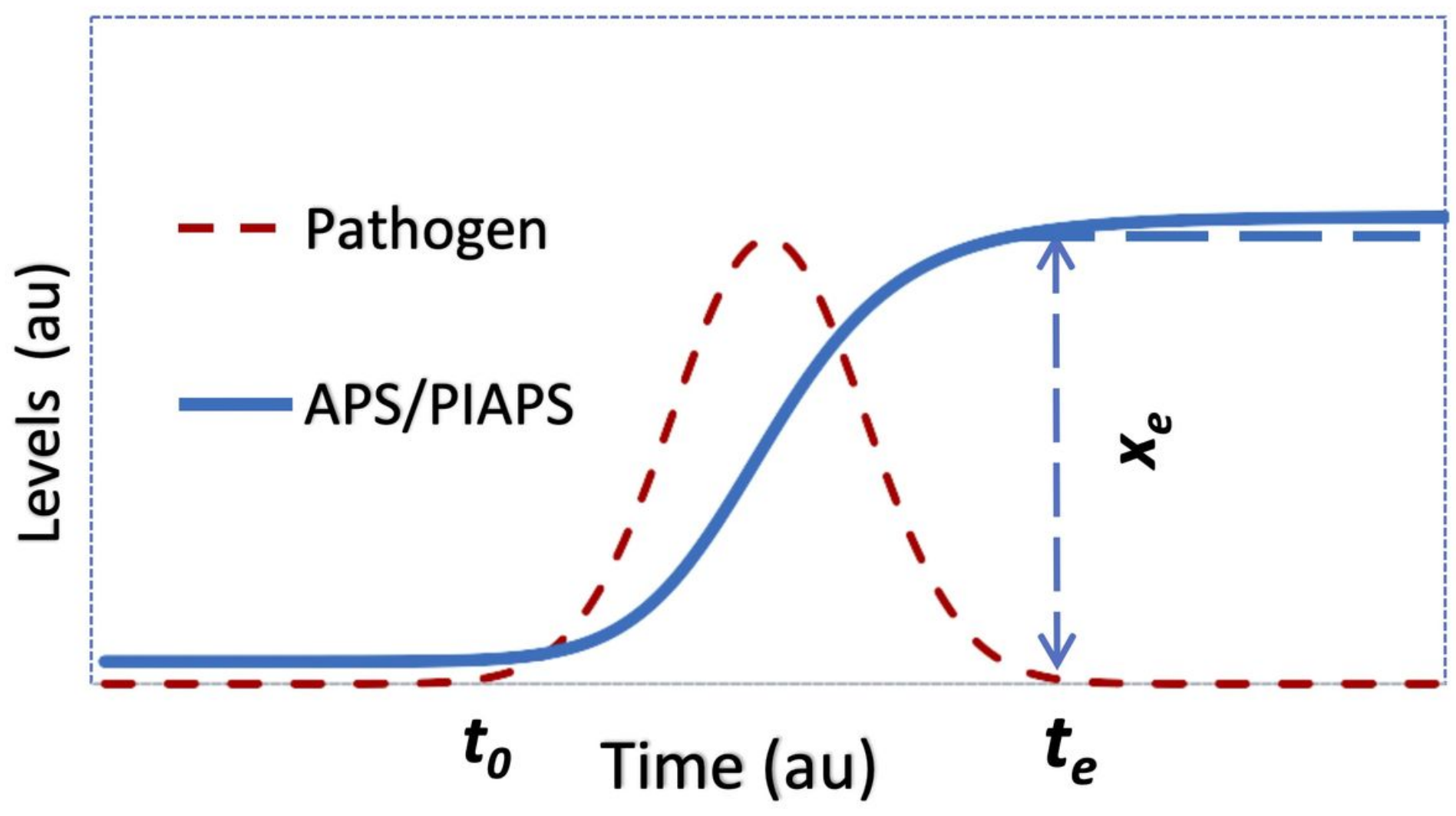

Figure 1

Schematic levels of pathogen (short dashed red curve) and host immune system generated antipathogen species (APS), including proinflammatory anti-pathogen species (PIAPS), for normal (solid blue curve) and abnormal (long dashed blue line, reflecting hyperinflammatory disorder) immune response reactions over time.

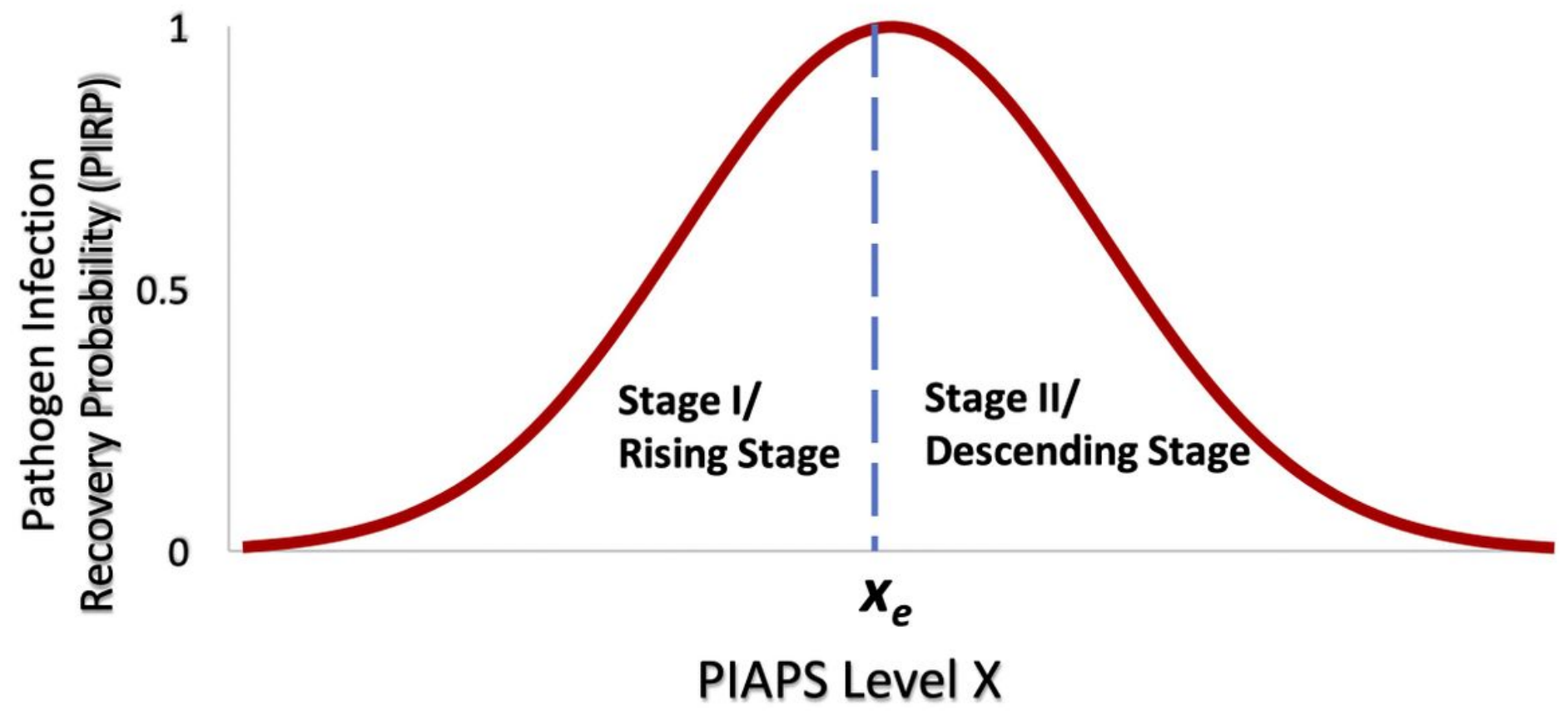


Figure 2

Scheme of Pathogen Infection Recovery Probability (PIRP) versus certain Proinflammatory Anti-Pathogen Species (PIAPS) levels based on equation 1.

\section{Supplementary Files}

This is a list of supplementary files associated with this preprint. Click to download.

- COVID19Comm031120.pdf 